Evaluation of resistive-plate-chamber-based TOF-PET applied to in-beam particle therapy monitoring

This content has been downloaded from IOPscience. Please scroll down to see the full text. 2015 Phys. Med. Biol. 60 N187

(http://iopscience.iop.org/0031-9155/60/9/N187)

View the table of contents for this issue, or go to the journal homepage for more

Download details:

IP Address: 157.193.227.128

This content was downloaded on 20/04/2015 at 12:09

Please note that terms and conditions apply. 


\title{
Evaluation of resistive-plate-chamber-based TOF-PET applied to in-beam particle therapy monitoring
}

\author{
I Torres-Espallardo ${ }^{1,6}$, F Diblen ${ }^{2,6}$, H Rohling $^{3,6}$, P Solevi $^{1}$, \\ J Gillam ${ }^{1,4}$, D Watts ${ }^{5}$, S España ${ }^{2}$, S Vandenberghe ${ }^{2}$, F Fiedler $^{3}$ \\ and $M$ Rafecas ${ }^{1}$ \\ ${ }^{1}$ Instituto de Física Corpuscular-IFIC (UV/CSIC), E-46071 Valencia, Spain \\ 2 Department of Electronics and Information Systems, MEDISIP, Ghent-University, \\ iMinds Medical IT-IBiTech, B-9000 Ghent, Belgium \\ ${ }^{3}$ Helmholtz-Zentrum Dresden-Rossendorf, D-01328 Dresden, Germany \\ ${ }^{4}$ Brain and Mind Research Institute-BMRI, Univesity of Sydney, Camperdown \\ NSW 2050, Australia \\ 5 TERA Foundation, I-28100 Novara, Italy \\ E-mail: irene.torres@uv.es
}

Received 13 August 2014, revised 24 February 2015

Accepted for publication 10 March 2015

Published 17 April 2015

\begin{abstract}
Particle therapy is a highly conformal radiotherapy technique which reduces the dose deposited to the surrounding normal tissues. In order to fully exploit its advantages, treatment monitoring is necessary to minimize uncertainties related to the dose delivery. Up to now, the only clinically feasible technique for the monitoring of therapeutic irradiation with particle beams is Positron Emission Tomography (PET). In this work we have compared a Resistive Plate Chamber (RPC)-based PET scanner with a scintillation-crystal-based PET scanner for this application. In general, the main advantages of the RPCPET system are its excellent timing resolution, low cost, and the possibility of building large area systems. We simulated a partial-ring scanner based on an RPC prototype under construction within the Fondazione per Adroterapia Oncologica (TERA). For comparison with the crystal-based PET scanner we have chosen the geometry of a commercially available PET scanner, the Philips Gemini TF. The coincidence time resolution used in the simulations takes into account the current achievable values as well as expected improvements of both technologies. Several scenarios (including patient data) have been simulated to evaluate the performance of different scanners. Initial results have shown that the low sensitivity of the RPC hampers its application to hadron-
\end{abstract}

\footnotetext{
${ }^{6}$ These authors contributed equally to the work.
} 
beam monitoring, which has an intrinsically low positron yield compared to diagnostic PET. In addition, for in-beam PET there is a further data loss due to the partial ring configuration. In order to improve the performance of the RPC-based scanner, an improved version of the RPC detector (modifying the thickness of the gas and glass layers), providing a larger sensitivity, has been simulated and compared with an axially extended version of the crystal-based device. The improved version of the RPC shows better performance than the prototype, but the extended version of the crystal-based PET outperforms all other options.

Keywords: PET, in-beam, RPC, particle therapy, TOF, range deviation, partial-ring

(Some figures may appear in colour only in the online journal)

\section{Introduction}

The number of particle therapy facilities for the treatment of cancer with proton or carbon ions (PTCOG 2014) is increasing worldwide. Particle therapy (PT) provides precise dose deposition and a sparing of normal tissue due to the favorable depth dose distribution of hadrons in matter. At the end of the particle range the specific energy loss of a particle energy shows a maximum (Bragg peak), producing dose distributions with high conformity. However, there are uncertainties resulting from patient positioning errors, conversions of attenuation coefficients from computer tomography (CT) to proton stopping power, intrafraction motion and inter-fraction anatomy changes. These uncertainties can compromise the benefits of PT and can lead to under-coverage of the tumor or delivery of extra dose to normal tissue (Paganetti 2012, Knopf and Lomax 2013). Non-invasive monitoring of the particle range is highly desired. When particles (protons, carbon ions) traverse the tissue, positron emitting isotopes are generated due to nuclear reactions. The posterior annihilation of the emitted positron is followed by two coincident photons traveling in opposite directions that can be detected using Positron Emission Tomography (PET). The distribution of the $\beta^{+}$ radioisotopes is related to the dose distribution of the particle beam. Although the relationship is not straightforward, this technique can be used for the quality control of the treatment and currently PET is the only clinically applied technique for PT monitoring (Oelfke et al 1996, Parodi and Bortfeld 2006, Parodi et al 2007, Min et al 2013, Remmele et al 2011). The feasibility of acquiring PET data during treatment (in-beam PET) was proven at GSI Helmholtzzentrum für Schwerionenforschung (Darmstadt, Germany) for stable carbon ions (Enghardt et al 2004). In Nishio et al (2006) the on-line monitoring of proton irradiation by PET was analyzed and Parodi et al (2007) proved by a clinical study that PET-CT measurements after proton irradiation are feasible. Other emerging techniques for treatment monitoring based on secondary radiation generated during the delivery, such prompt photons and protons, which are the product of nuclear reactions are beyond the scope of this article.

When the PET acquisition is performed within the treatment room during or directly after one fraction of the therapeutic irradiation, the PET technique is named in-beam and in-room, respectively. If the PET acquisition is done out of the treatment room (usually 10-30 min after the irradiation), it is referred to as off-line or off-beam PET. Off-line PET suffers from a further reduction of activity due to two factors. On the one hand, the isotopes produced during treatment have a short half-life (few seconds to $20 \mathrm{~min}$ ) so that a large fraction of $\beta^{+}$decays 
occurs during treatment. On the other hand, biological wash-out spreads most of the generated isotopes away from the irradiated volume. When using in-beam PET, the reconstructed images are closer to the original $\beta^{+}$distribution than the images obtained from in-room and off-line acquisitions since physical decay and wash-out are minimized. In Shakirin et al (2011) it is demonstrated that in-beam PET is the best method in terms of count rate.

The challenge of in-beam PET is the integration of the device into the treatment site. In order to avoid collisions between the beam and the PET scanner, a partial-ring geometry (Enghardt et al 2004), a dual-ring OpenPET geometry (Yamaya et al 2008) or a tilted singlering (Tashima et al 2012) are required. For this work, the partial-ring configuration is the preferred option. The limited angular coverage can lead to data truncation of the reconstructed images and elongation artifacts. An explanation of these artifacts applied to hadrontherapy monitoring with partial-ring configuration can be found in Crespo et al (2006). To mitigate the latter, Time-of-Flight (TOF) information can be integrated into the reconstruction algorithm (Shakirin 2009, Surti et al 2011). For image reconstruction, the Maximum Likelihood Estimation Maximization (MLEM) is a commonly used method for PET and provides the possibility to include TOF information (Groiselle and Glick 2004). The improvement in image quality achieved by TOF-MLEM reconstruction compared with standard MLEM for PET strongly depends on the time resolution of the scanner. Therefore, PET technologies with exceptional time resolution are specially suited for in-beam monitoring. The Resistive Plate Chambers (RPCs) are gaseous, parallel plate detectors working in avalanche mode whose imaging capabilities make them interesting for PET applications (Blanco et al 2009). The main advantages of RPC technology are excellent timing resolution, inexpensive construction over large areas and the capability to provide Depth Of Interaction (DOI) information, due to a highly layered structure. RPCs provide a better time resolution (Blanco et al 2003) than inorganic scintillating crystals, commonly used in PET. In the case of $511 \mathrm{keV}$ photons the time resolution obtained with these detectors is $\sigma=90 \mathrm{ps}$ (Blanco et al 2003), which would lead to 300 ps FWHM coincidence resolving time), whereas for commercially available crystal-based PET scanners the best coincidence resolving time (CRT) is currently $\approx 500-600$ ps FWHM. As an example, the Philips Gemini TF made of LYSO and used for diagnostic PET shows 585 ps FWHM CRT (Surti et al 2007). Recently, a new generation of PET scanners based on silicon photomultiplier provides CRTs of $400 \mathrm{ps,} \mathrm{as} \mathrm{referenced} \mathrm{in} \mathrm{manufacturer} \mathrm{data} \mathrm{sheets}$ (Philips Vereos PET/CT ${ }^{7}$, GE Signa PET/MR ${ }^{8}$ ).

In this manuscript, the RPC-technology for in-beam PET particle therapy monitoring is studied in detail and compared to a well-established PET detector technology, based on scintillating crystal. The commercially available TOF-capable Philips Gemini TF has been used as a basis for the geometry of a reference crystal-based PET-scanner. The constrains posed by the low sensitivity of the RPC scanner could be overcome through its excellent timing resolution, by building large area detectors and increasing the number of stacks. With regard to the image reconstruction, the low sensitivity can be partially compensated using TOF-MLEM (Conti 2009). Finally, when working with the beam on, a high number of random coincidences is expected in both systems, but being the random rate a function of the singles and the timing window it might affect the RPC scanner in a lesser extent. All these advantages combined in one technology could make that a gas-based detector system such as RPC could compete favorably with the well-established crystal-based technology for PET. For this purpose, the RPC prototype, dedicated to in-beam PET, that is under construction at the TERA foundation

\footnotetext{
${ }^{7}$ http://incenter.medical.philips.com/doclib/enc/10797631/452299106961_VereosBrochureREVISE_FNL_ lr.pdf $\% 3$ ffunc\%3ddoc.Fetch\%26nodeid\%3d10797631\%26vernum\%3d1

${ }^{8}$ www3.gehealthcare.com/en/products/categories/magnetic_resonance_imaging/signa_pet-mr\#tabs/tab2892C34FC72C4A0497D37371F0D391EA
} 
(TERA 2013) was chosen as a starting point. An improved RPC-scanner was also considered which used a modified geometry in order to increase sensitivity. All the crystal-based and RPC-based scanners included in our study are considered with their currently achievable time resolutions and improved time resolutions which take into account future developments.

In order to fulfill the goals of this investigation, simulation studies of sensitivity, spatial resolution and scatter fraction for both technologies and different configurations were conducted. Results concerning the abilities of each system to detect deviations of particle range are provided. Finally, the scanners were compared with respect to the quality of reconstructed images using real patient data.

In Crespo et al (2012), both technologies are also compared for diagnostic purposes with NEMA and anthropomorphic phantoms. But the geometry proposed by those authors for the RPC-based system is not feasible for our task, and as such we have adapted the system for online treatment monitoring. In addition, the characteristics of the sources obtained in particle therapy monitoring are different than the ones used in diagnostic scenarios. To our knowledge this is the first work that compares thoroughly both technologies in the same manuscript with a focus on beam monitoring in hadrontherapy with phantoms and patient data.

\section{Materials and methods}

In this study we compare the use of RPC-based and crystal-based PET scanners with partialring configuration for in-beam monitoring of hadron therapy treatments. In the first part of this section, the geometries and the main characteristics of the two scanner technologies are introduced. Since the generation of the PET signal for both technologies is intrinsically different, a second part in this section is dedicated to the simulations of the acquisition of the PET signal. For the evaluation of the capabilities of such systems we have employed several source distributions to obtain a general view of the scanner response and a specific view for the given task (treatment monitoring). The preparation of all sources is described in the third part of this section. The last two parts provide an explanation of the reconstruction algorithm employed and the tools for the quantification of the reconstructed images, respectively.

\subsection{Scanners under investigation}

In order to have reference performance values we included PET systems with full-ring configuration in our comparison. Initially, standard axial dimension of around $18 \mathrm{~cm}$ was employed in the crystal-based PET, whereas the axial dimension of the RPC-prototype developed at TERA was set to $30 \mathrm{~cm}$ to compensate for its low sensitivity. One difference of this application to diagnostic PET is that the induced activity is low $\left(6600 \mathrm{~Bq} \mathrm{~Gy}^{-1} \mathrm{~cm}^{-3}\right.$ for ${ }^{1} \mathrm{H}, 1600$ $\mathrm{Bq} \mathrm{Gy}^{-1} \mathrm{~cm}^{-3}$ for ${ }^{12} \mathrm{C}$ (Priegnitz et al 2008)) and and a fraction of the produced isotopes have shorter half lives significantly reducing the activity in inter-spill and post-treatment acquisition modalities. Another difference is that to reduce the effect of the short lived isotopes and wash-out, the whole acquisition should be performed in one bed position that covers the treatment area sufficiently. In addition, the use of a partial-ring configuration leads to count losses. To address these difficulties, both technologies were additionally simulated with an extended axial dimension. In the case of the RPC, a further improvement in terms of detector radial depth was also introduced to gain efficiency.

2.1.1. RPC-based PET. This system is based on a prototype developed by the TERA group TERA (2013). The detector head is made up of RPC stacks (figure 1). One stack consists of 

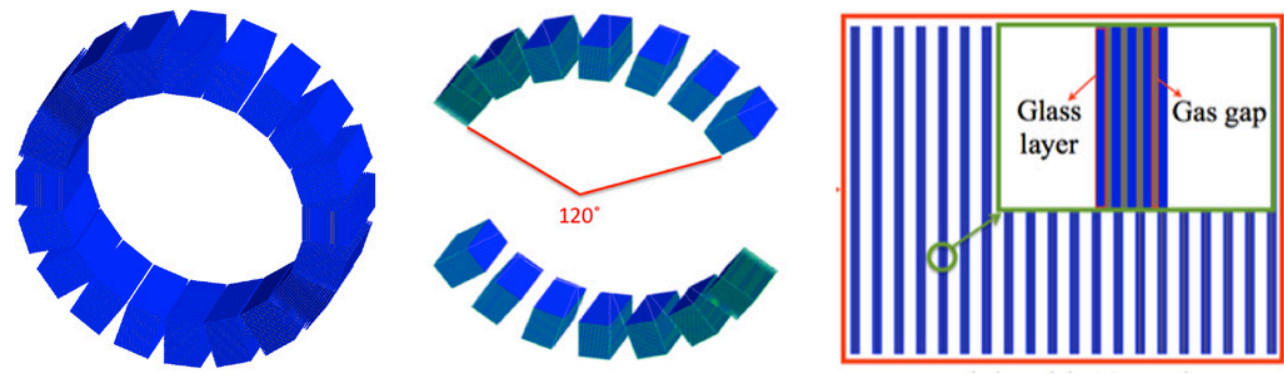

Figure 1. Schema of the RPC-based system for full-ring (left) and partial-ring (center), and a zoom of one head with 20 stacks (right) to show the glass-gap layered structure.

five parallel glass plates $400 \mu \mathrm{m}$ thick separated by $300 \mu \mathrm{m}$ thick gas gaps filled with Freon leading to a stack size of $3.2 \times 120 \times 300 \mathrm{~mm}^{3}$ (indicating the radial, transverse and axial dimensions of a stack respectively). An air gap of $4.8 \mathrm{~mm}$ was defined between adjacent stacks, providing an $8 \mathrm{~mm}$ pitch. Each stack has independent readout electronics able to identify the interaction location with a spatial resolution of 2 and $4 \mathrm{~mm} \sigma$ in the transversal and axial directions, respectively. The DOI can be recovered by identifying in which stack the photon interacted. Each stack is readout with electrodes at each end, then an encoding is made in firmware for each stack. A comparison of scanners based on different numbers of stacks (20, 40 and 60) per detector head was accomplished in order to identify the optimal number. The full-ring (FR) scanner configuration consists of 20 detector heads arranged in a ring with a diameter of $80 \mathrm{~cm}$ and $30 \mathrm{~cm}$ axial dimension. For the partial-ring (PR) scanner the number of detector heads was reduced to 14 (in-plane angular coverage of $120^{\circ}$ ). With this angular coverage, the device has two open regions of $60^{\circ}$ each with a $40 \mathrm{~cm}$ aperture. This arrangement avoids collisions with the nozzle during beam delivery. In addition, the PET device would not be damaged by the outgoing fragments, a high percentage of which have forwardpeaked distributions with maximum angles of $10^{\circ}$ in the case of carbon-ion therapy (Hättner et al 2013). The extended and improved partial-ring configuration was obtained by increasing the axial extent of the scanner to $60 \mathrm{~cm}$ and reducing the glass thickness and air gap. The efficiency of an RPC-module depends on the conversion probability in the glass layer of the 511 $\mathrm{keV}$ photon to an electron and of the emerging probability of the resultant electron from the glass layer into one of the gas gaps. The design of the module is a trade-off between these two probabilities. The thicker the glass layer, the higher the conversion probability, but as a result the emerging probability is lower. A simulation study was performed to provide the optimum value for the thickness of the glass layer and gas gaps (Watts 2014). Each stack was modified by downsizing the thickness of the glass plates to $150 \mu \mathrm{m}$ and the air gaps between stacks to $2.05 \mathrm{~mm}$. In this way, the stack size was $1.95 \times 120 \times 600 \mathrm{~mm}^{3}$. For this configuration we only simulated detector heads of 60 stacks. This configuration will be referred to as improved. Table 1 shows the design parameters of the RPC-based PET scanners under investigation. The TERA group reported an achievable CRT for the RPC module of 200 ps (FWHM). While this CRT is yet to be proven, there is still an expectation room for improvement (Watts et al 2013, Watts 2014). In our study we compared the performance of RPC-based PET systems with 50, 100 and 200 ps (FWHM) CRT. Although there are no experimental results validating these values, we have considered that for this comparison study the time resolution values of the RPC-based must be much better than the crystal-based system in order to compensate for the low sensitivity. In Watts (2014) is claimed that $150 \mathrm{ps}$ is realistically achievable. In the following, all the values that refer to CRT are expressed in terms of FWHM. 
Table 1. Design parameters of the RPC-based PET scanners.

\begin{tabular}{llll}
\hline & & \multicolumn{2}{c}{ Partial-ring } \\
\cline { 3 - 4 } & Full-ring & Standard & Improved \\
\hline Axial Detector Size $(\mathrm{mm})$ & 300 & 300 & 600 \\
Number of heads & 20 & 14 & 14 \\
Number of stacks per head & $20,40,60$ & $20,40,60$ & 60 \\
Glass thickness $(\mu \mathrm{m})$ & 400 & 400 & 150 \\
Stack thickness $(\mathrm{mm})$ & 3.2 & 3.2 & 1.95 \\
Air gap $(\mathrm{mm})$ & 4 & 4 & 2.05 \\
\hline
\end{tabular}

2.1.2. Crystal-based PET. The simulated scanners are based on the geometry and timing characteristics of the Philips Gemini TF PET (Surti et al 2007, Karp et al 2008). The full-ring configuration contains 28 detector heads distributed in a ring with a diameter of $90.34 \mathrm{~cm}$ and an axial dimension of $17.92 \mathrm{~cm}$. Each detector module consists of $23 \times 44$ LYSO crystals with an individual size of $4 \times 4 \times 22 \mathrm{~mm}^{3}$. The simulated crystal-based systems are shown in figure 2 . The standard partial-ring configuration comprises 20 heads with $120^{\circ}$ in-plane angular coverage each (figure 2). The extended partial-ring configuration was obtained by increasing the number of crystal rows on each detector head from 44 to 98 in order to achieve a 39.92 $\mathrm{cm}$ axial coverage. This extended version is approximately equivalent to the improved RPCmodule. Table 2 summarizes the design parameters of the crystal-based PET scanners under investigation. An energy resolution of $11.5 \%$ and an energy window of $440-665 \mathrm{keV}$ were used for all simulations. In terms of CRT, current commercial TOF-PET scanners already provide 585 ps FWHM (Surti et al 2007). In addition, recent studies (van Dam et al 2013) have shown that it is possible to reduce the CRT below $200 \mathrm{ps}$. Therefore, we compared the performance of systems with CRTs of 200, 400 and 600 ps.

\subsection{Simulation of PET systems}

Acquisitions of the crystal-based and RPC-based PET systems were simulated using the GATE simulation toolkit (Jan et al 2011), version 6.1.

2.2.1. RPC-based PET. In an RPC detector, an incoming $511 \mathrm{keV}$ photon interacts in the glass plates where a secondary electron is generated. A high differential voltage is applied to electrodes placed on both sides of each stack in order to accelerate the electrons and produce a cascade. Only when secondary electrons escape from the glass and reach the gas layer with enough energy, avalanche multiplication is initiated. In order to model this behavior within GATE the internal gas layers in the stacks are defined as sensitive volumes. In that way, a detection is only triggered when a particle deposits sufficient energy in one of the sensitive volumes. The readout of the RPC-based scanner is conducted at the level of the stack. To reproduce this readout in the simulations, the singles are generated after adding together hits produced in a stack. The singles were stored from the GATE simulation and the postprocessing was done using software developed in-house. The post-processing code performs the spatial and time blurring, as well as coincidence sorting. No multiple events (more than 2 singles within a time coincidence window) are considered in this study. For the physical models of the simulations of the RPC stacks, the Penelope electromagnetic model was used and secondary particles with a range smaller than $1 \mu \mathrm{m}$ (range cut) were not tracked.

In order to validate the simulations, the RPC-prototype developed at TERA was simulated and the results obtained were compared with experimental data (Watts et al 2013). In the 

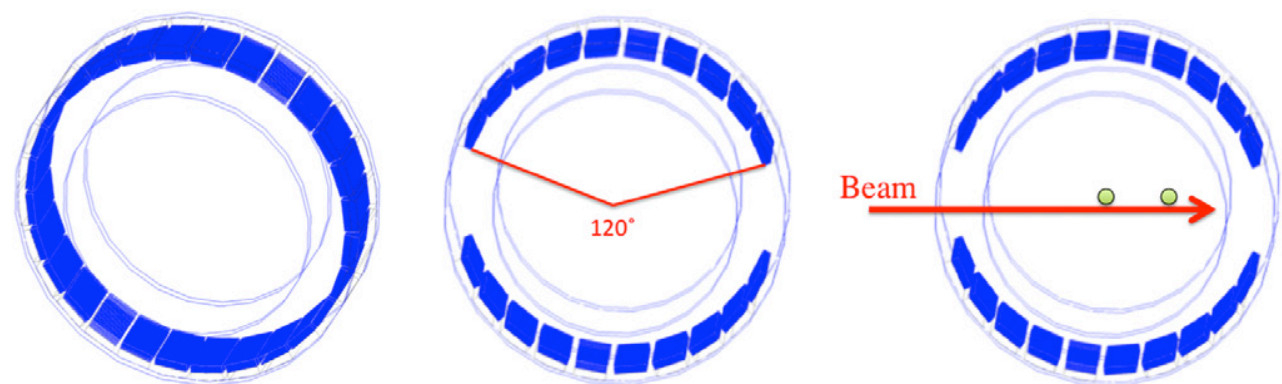

Figure 2. Schema of the crystal-based system for full-ring (left) and partial-ring (center) configurations. Schematics of the setup employed to study the spatial resolution (right). The direction of a typical beam is shown by the red arrow. The circles represent the location of the point-like sources

Table 2. Design parameters of the crystal-based PET scanners.

\begin{tabular}{lllr}
\hline & & \multicolumn{2}{c}{ Partial-ring } \\
\cline { 3 - 4 } & Full-ring & Standard & Extended \\
\hline Axial Size $(\mathrm{mm})$ & 179.2 & 179.2 & 399.2 \\
Crystals per head & $23 \times 44$ & $23 \times 44$ & $23 \times 98$ \\
Number of heads & 28 & 20 & 20 \\
\hline
\end{tabular}

simulations, a collimated ${ }^{22} \mathrm{Na}$ source was placed at the center between two heads and the singles detection efficiency was computed. In order to reproduce the measured efficiency we adjusted the minimum energy that the secondary electron needed to deposit in the gas in order to trigger an avalanche.

2.2.2. Crystal-based PET. In crystal-based scanner simulations, the annihilation photon deposits energy in the scintillator material and the amount of deposited energy is processed by the digitizer module. The digitizer module generates singles depending on the readout parameters. Although individual crystal readout is not currently standard in clinical scanners, we have considered this option. Individual crystal coupling represents a lower sensitivity scenario, it may also enhance the detection resolution and it provides best timing performance (Auffray et al 2013). Moreover, it is the option in the new Philips VEREOS system with digital silicon-photomultipliers. The whole acquisition chain (from hits to coincidences), except time blurring, was simulated using GATE modules, from the energy response (energy resolution and energy window) to the coincidence selection. No multiple events were considered. The simulation of the crystal-based scanner was validated using the results found in Surti et al (2007), taking into account the individual crystal readout of the simulated system. The Penelope model was also used and the range cut for secondary particles was set to $0.1 \mathrm{~mm}$.

The specific CRT of each scanner technology is added after simulation and before image reconstruction. Table 3 lists the parameters used in the simulations and in the post-processing prior to image reconstruction.

\subsection{Simulated sources}

2.3.1. Performance studies. We have evaluated the performance of both technologies following the NEMA NU-2 2001 protocol (NEMA 2001). Although this protocol is dedicated 
Table 3. Parameters for simulations and coincidence sorting.

\begin{tabular}{lll}
\hline & Crystal-based & RPC-based \\
\hline Energy Resolution & $11.5 \%$ & - \\
Energy Window & $440-665 \mathrm{keV}$ & $>100 \mathrm{eV}$ \\
Spatial Blurring (mm) & - & $\sigma_{y}=2, \sigma_{z}=4$ \\
Coincidence Time Window (ns) & 3.8 & 1.0 \\
Coincidence Resolving Time & $200,400,600$ & $50,100,200$ \\
(FWHM, ps) & & \\
\hline
\end{tabular}

to clinical PET scanners, it was used due to the lack of a specific protocol for PT monitoring. From the protocol, we selected tests that correspond to basic intrinsic measurements like sensitivity, spatial resolution and scatter fraction. The analysis of the sensitivity is a key parameter in PET and is also specifically important in PT, since the expected activity levels are one order of magnitude lower than clinical PET. The spatial resolution characterizes the ability of a system to distinguish two points. This magnitude should reflect the ability to determine the distal edge of the activity distribution. Finally, we have studied the scatter fraction since the RPC-based PET has no energy information, necessary to discriminate scatter events. For a more elaborated method to assess the system response applied to hadrontherapy monitoring, the authors refer to Crespo et al (2006).

Sensitivity. The absolute sensitivity of each scanner was estimated by using a $15 \mathrm{~cm}$ long line source and a point source with $1 \mathrm{MBq}$ of activity each. The two sources were situated at the axial center and with a radial offset of $10 \mathrm{~cm}$ (in $x$-direction). We used a back-to-back gamma source in GATE to speed simulations and to prevent positrons from escaping (source is placed in air). Although simulating the source within a water phantom could represent a more realistic scenario, it would not provide any additional information in this comparison study, while the simulation time would increase substantially due to the additional generation and tracking of positrons. The sensitivity was calculated as the ratio between the obtained rate of true coincidences and the activity of the source. Additionally, for the standard crystal-based system, a $70 \mathrm{~cm}$ long centered line source was also simulated to compare with published results (Surti et al 2007).

Spatial resolution. The spatial resolution was obtained using two point-like sources, one at the center of the FOV and another one with $10 \mathrm{~cm}$ radial offset towards one ring opening, both in the central slice of the scanner (figure 2). For this study we consider that range measurements are conducted in the beam direction along the ring opening. In a clinical scenario, this would not be always the case, since the orientation of the beam will depend on the location of the tumor. However, the selected locations provide the necessary information about the spatial resolution for comparison purposes. A sphere of $0.8 \mathrm{~mm}$ diameter filled with $10 \mathrm{MBq}$ of ${ }^{18} \mathrm{~F}$ covered by $0.2 \mathrm{~mm}$ thick glass was used for the assessment. $10^{6}$ events were recorded for each scanner configuration. From the reconstructed images, the FWHM of the point spread functions (PSFs) were determined in radial, tangential and axial dimensions following the NEMA protocol.

Scatter fraction. The scatter fraction (SF) was obtained using a Polymethyl Methacrylate (PMMA) cylinder with $20.3 \mathrm{~cm}$ diameter and $70 \mathrm{~cm}$ length, and an axially oriented line source filled with $10 \mathrm{MBq}$ of ${ }^{18} \mathrm{~F}$ with a radial direction offset of $4.5 \mathrm{~cm}$. The $\mathrm{SF}$ was calculated as the ratio between the scattered coincidences and the true plus scattered coincidences. 


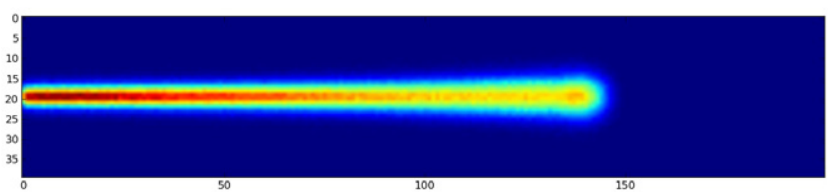

Figure 3. Activation map generated from a simulated proton beam of $160 \mathrm{MeV}$. The axes are in $\mathrm{mm}$.

2.3.2. Proton pencil beam study. The range deviation expected for different scanner configurations was evaluated by irradiation of a cylindrical PMMA phantom with an ideal proton pencil beam (neither spatial, nor energy spread were simulated). Although a single pencil beam does not represent a realistic scenario, the generated activity sources are a useful tool to analyze the effects of the partial-ring geometry and the TOF information. For that purpose we simulated the proton beam irradiation using Geant4 9.3 (Agostinelli et al 2003, Allison et al 2006) and the distribution of $\beta^{+}$-isotopes produced were recorded. Other secondary particles produced by the proton beam, like prompt gamma and neutrons were not taken into account. The $\beta^{+}$-isotope distribution was later used as an input source for the simulation of the different scanner configurations using GATE.

For the proton beam simulations, the physics list selected was the $Q G S P \_B I C \_H P$ for hadronic models with standard electromagnetic physics. Several proton beams of different energies were simulated. The simulated proton energies were chosen to provided a $3 \mathrm{~mm}$ range difference between consecutive energy values, from 156 to $162 \mathrm{MeV}$. The cylindrical PMMA cylindrical target was $20 \mathrm{~cm}$ in height and $15 \mathrm{~cm}$ in diameter. The target was oriented with its central axis parallel to the beam direction and its center situated at the origin. In this way, the beam hits the target at the center of one of the lateral sides. The acquisition time for the measurement was $30 \mathrm{~min}$ and the number of emitted protons was $5 \cdot 10^{7}$. In order to identify the $\beta^{+}$isotopes, we recorded the positrons that were produced by a radioactive decay. The source was then built by histograming the emission coordinates of the selected positrons (the positron range was not included). Only positrons emitted during the irradiation time and the following 30 min were considered. This simplified approach is justified as it is to be used only in a comparison study. It is important to mention that the acquisition time in a clinical inbeam PET application would probably be shorter. But for testing the capabilities of the RPC technology, $30 \mathrm{~min}$ were considered appropriate as an upper limit. The generated source for a $160 \mathrm{MeV}$ proton beam is shown in figure 3, and the number of generated positrons included in the acquisition of $30 \mathrm{~min}$ can be seen in table 4 for each beam energy.

The reconstructed images of these simulated acquisitions were used to evaluate the ability of each scanner configuration to detect range shifts by measuring the distal edge of the reconstructed distributions, as explained in section 2.5.

2.3.3. Patient data study. In order to evaluate the image quality of the scanner configurations in a realistic scenario, we used data from one head-and-neck patient randomly chosen from those treated with carbon ions at GSI facility (GSI Helmholtzzentrum für Schwerionenforschung Darmstadt, Germany). The patient was suffering from a chordoma and was treated with $0.829 \mathrm{~Gy}$ in a unique field in each fraction.

An in-house existing code (Pönisch et al 2004), that calculates the activation of patient tissues, was used to simulate patient irradiation according to the real treatment plan and CT images obtained from GSI. The outcome of this code is a 3D map of the positron annihilation coordinates. For this patient, we created a 3D map with the annihilation coordinates of the 
Table 4. Simulated proton beam energies, ranges and generated positrons in $30 \mathrm{~min}$ acquisition.

\begin{tabular}{lllll}
\hline Energy $(\mathrm{MeV})$ & Range $(\mathrm{cm})$ & $\begin{array}{l}\text { Number of } \\
\mathrm{e}^{+} \text {in 30 min. }\end{array}$ & R50 $(\mathrm{mm})$ & RCD $(\mathrm{mm})$ \\
\hline 156.4 & 14.6 & $2.2 \cdot 10^{6}$ & 137.7 & 135.4 \\
158.2 & 14.9 & $2.2 \cdot 10^{6}$ & 140.6 & 138.2 \\
160.0 & 15.2 & $2.3 \cdot 10^{6}$ & 143.5 & 141.1 \\
161.9 & 15.5 & $2.3 \cdot 10^{6}$ & 146.7 & 144.2 \\
163.6 & 15.8 & $2.4 \cdot 10^{6}$ & 149.4 & 146.9 \\
\hline
\end{tabular}

Note: In the last two columns, calculated $R 50$ and $R C D$ for original activation maps for the different proton beam energies.

positrons emitted by the ${ }^{15} \mathrm{O},{ }^{14} \mathrm{O},{ }^{13} \mathrm{~N},{ }^{11} \mathrm{C}$ and ${ }^{10} \mathrm{C}$ isotopes produced during the irradiation. As in the real case, only the positrons which would annihilate during the extraction pauses of the synchrotron were included. Furthermore, the decay of the aforementioned nuclides, and the propagation and annihilation of the positrons is handled by the software. Since the algorithm modeled the activation maps, other secondary particles, like prompt-gamma and neutrons, that could generate random coincidences were not simulated. Nevertheless, the contribution of the prompt-gamma in this scheme is strongly minimized because the acquisition takes place during the extraction pauses. The neutron contribution is also expected to be negligible. The simulated maps of annihilation points were centered at the isocenter and used as a source in the GATE simulations for each scanner configuration. Figure 4 shows the dose distribution according to the treatment plan. All images are superimposed on the corresponding CT images.

Finally, the reconstructed images obtained by the simulated PET acquisitions were compared to the predicted simulation results. For this work, the comparison was done qualitatively by visual evaluation and by the analysis of selected profiles along the beam direction.

\subsection{Image reconstruction}

Simulated data were reconstructed using a 3D list-mode MLEM reconstruction algorithm including TOF:

$$
f_{j}^{m+1}=\frac{f_{j}^{m}}{\sum_{i=1}^{I} p_{i j}} \sum_{i=1}^{I} p_{i j, \delta T} \frac{1}{\sum_{b=1}^{J} p_{i b, \delta T} f_{b}}
$$

where $f_{j}^{m+1}$ is the unknown activity distribution at the $m+1$ th iteration, discretized into $J$ voxels. There are $I$ recorded coincidences in list-mode data and each is characterized by a time difference $\delta T$. Finally, $p_{i j, \delta T}$ is proportional to the probability that an emission produced in voxel $j$ is detected in Line-Of-Response (LOR) $i$ with a time difference of $\delta T$. These probabilities were calculated using Siddon ray-tracing algorithm (Siddon 1985). In the case of the patient data, the system matrix was assessed using a code developed specifically for this application (Laukner 1999) and the sensitivity matrix was calculated in advance. The time information was modeled within $p_{i j}, \delta T$ by weighting each LOR by a Gaussian distribution with FWHM equal to the CRT and mean value equal to the expected position of the emission using $\delta T$ (Groiselle and Glick 2004). The field-of-view of the crystal-based scanners was kept as standard: $57.6 \times 57.6 \times 18 \mathrm{~cm}^{3}$, despite the axial extension. In the case of the RPC 

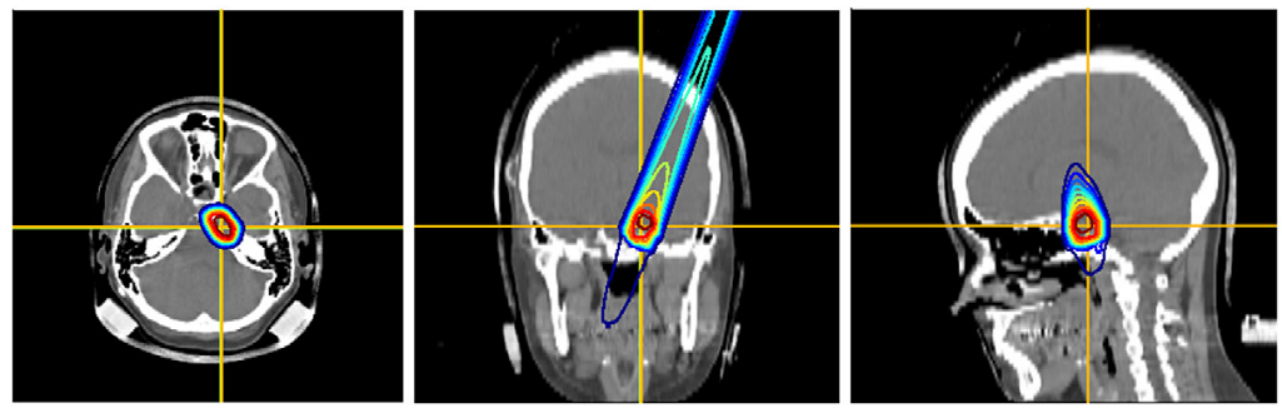

Figure 4. Axial, coronal, and sagittal planned dose distribution for the patient.

system, similar sizes were used, $40 \times 40 \times 20 \mathrm{~cm}^{3}$. Since it is a comparison study between two technologies and the feasibility of using RPC technology for hadrontherapy monitoring must be determined, we will work with the cleanest type of coincidences. In addition, in order to count for random and scatter coincidences the simulations would require a higher level of details in the electronic modeling that are out of the scope of this work and some important answers already come from the simplified scenario. Therefore, only true coincidences were used for reconstruction, random and scatter coincidences were removed from the prompt data by using information provided by the simulations. For reconstruction of the point sources for spatial resolution evaluation and positron distributions generated by a proton pencil beam, a voxel size of $1 \times 1 \times 1 \mathrm{~mm}^{3}$ was employed. This voxel size was necessary to assess the spatial resolution ${ }^{9}$ and here we attempt to differentiate a $3 \mathrm{~mm}$ range shift. In the case of patient data, the voxel size of the reconstructed image was larger $\left(2 \times 2 \times 2 \mathrm{~mm}^{3}\right)$, in order to reduce the noise resulting from the low number of events during therapeutic irradiation with ${ }^{12} \mathrm{C}$ and to keep a voxel size that allow us to visualize small details (Shakirin et al 2008).

\subsection{Quantitative analysis for distal edge estimation in proton pencil beams}

In order to assess the distal edge of activation maps from the reconstructed beam-like sources, two figures-of-merit have been used. The distal edge at $50 \%(R 50)$ of the maximum intensity and at $98 \%$ of the cumulative distribution $(R C D)$ along the beam were applied. The cumulative distribution of the reconstructed activity along the beam was employed in order to reduce the effect of the image noise in the calculation of the distal edge. While determination of the $R 50$ point may be ambiguous in noisy conditions the cumulative distribution guarantees a monotonically increasing profile for a reconstructed image with non-negative values. The $98 \%$ point was chosen empirically based on the simulation conditions: $3 \mathrm{~mm}$ between the ranges of adjacent beams using the simulated emission distributions. For the calculation of both quantities a region-of-interest (ROI) centered on the beam axis ( $1 \mathrm{~cm}$ wide) was employed. The width of the ROI was taken considering that the lateral spread of protons for the simulation energies in PMMA is around $1 \mathrm{~cm}$. For an estimation of the error of these quantities, the corresponding value of the simulated source distribution was calculated and used as a reference (see table 4). Since the main goal of proton therapy monitoring is to detect range deviations, the differences between the calculated range and the range of a reference energy $(160 \mathrm{MeV})$ was evaluated to study the verification capabilities of each system. Proton beam energies were

\footnotetext{
${ }^{9}$ According to NEMA protocol the pixel size should be one-third of the expected FWHM
} 
chosen to reproduce $3 \mathrm{~mm}$ differences in range, so that when comparing with the reference energy of $160 \mathrm{MeV}$, the distal edge deviations should ideally be \pm 3 and $\pm 6 \mathrm{~mm}$. It is important to note that the reconstructed images are produced from ideal data since no spurious events (random coincidences or scatter coincidences) were taken into account.

\section{Results}

\subsection{Validation of the efficiency of RPCs}

Since our detection model of the RPC was simplified and did not take into account the avalanche dynamics, we first validated our simulations with experiments. The experimental value of the singles efficiency $(0.66 \%)$ measured by the TERA group was used for validation (Watts et al 2013). In the experimental setup, a 5-layer (400 $\mu \mathrm{m}$ glass) stack was situated opposite a $2.54 \mathrm{~cm}$ long BGO crystal coupled to a photomultiplier and a ${ }^{22} \mathrm{Na}$ point-like source was placed in the center to measure the efficiency. In Monte Carlo simulations, the efficiency was assessed by using two opposite stacks of 5 glass layers while limiting the emission angle of the point source in order to reproduce similar values as the experiment. We have performed two different simulations. A collimated source of $511 \mathrm{keV}$ back-to-back photons situated in the center of both modules. To evaluate the effect of the $1.275 \mathrm{MeV}$ photon emitted by the ${ }^{22} \mathrm{Na}$, additional simulations were conducted adding an extra source consisting of $1.275 \mathrm{MeV}$ photons emitted isotropically. When the lower energy threshold is set at $125 \mathrm{eV}$, the efficiency obtained in the simulations is equal to the experimental value. If only $511 \mathrm{keV}$ photons are taken into account ${ }^{10}$, the experimental efficiency is reproduced at a threshold of $100 \mathrm{eV}$. For the following simulations $100 \mathrm{eV}$ was taken as the low energy discriminator.

\subsection{Performance Studies}

3.2.1. Sensitivity. The results for the sensitivity obtained using $15 \mathrm{~cm}$ line and point sources at different locations are shown in table 5 for each scanner configuration. The sensitivity obtained with the $70 \mathrm{~cm}$ centered line source for the standard full-ring crystal-based system is $4.01 \mathrm{cps} \mathrm{kBq}^{-1}$, lower than the experimental result reported by Surti et al (2007). This outcome is expected since we are simulating individual crystal readout which reduces sensitivity in comparison to systems which sum the signal of several crystals when forming coincidences. With the individual crystal readout, a signal is not recorded if the energy deposited by the photon within that crystal is below the threshold. With a block-detector readout, the signals for neighboring crystals are summed, and the position is assigned to the weighted average. Events that in the case of the individual crystal readout would be below the threshold (and thus rejected), in the block-detector might produce one single. However, for this comparison study, we opted for individual readout, since this scheme would improve the performance of the crystal-based scanner, and it is a feasible alternative given new photo-detectors' technology. For the crystal-based systems, the sensitivity of partial-ring configurations is $30 \%$ smaller than for full-ring scanners. With regard to the extended crystal-based configuration, as expected, the increase of the axial dimension is translated in a higher sensitivity of around $200 \%$ for the $15 \mathrm{~cm}$ line source and $100 \%$ for the point-like source. In the case of RPC-based systems, the sensitivity raised by $100 \%$ and $10-15 \%$ when increasing the number of stacks from 20 to 40 and from 40 to 60 , respectively when using a centrally located line source. This behavior is due to the shielding effect of the $400 \mu \mathrm{m}$ glass layers, almost $90 \%$ for 60

\footnotetext{
${ }^{10}$ The $1.275 \mathrm{MeV} \gamma$-rays emitted by the ${ }^{22} \mathrm{Na}$ source are not considered
} 
Table 5. Sensitivity in $\mathrm{cps} / \mathrm{kBq}$ for the studied sources, and PET system configurations.

\begin{tabular}{llllrrr}
\hline & & & & & Sensitivity $\left(\mathrm{cps} \mathrm{kBq}^{-1}\right)$ \\
\cline { 5 - 7 } Scanner & & & & & & Partial-ring \\
\cline { 5 - 7 } & Stacks & Position & Source & Full-ring & Standard & Extended/Improved \\
\hline Crystal-based & - & Center & Line-15 & 17.7 & $\mathbf{1 2 . 6}$ & 38.1 \\
RPC-based & 20 & Center & Line-15 & 2.2 & 1.6 & 4.5 \\
RPC-based & 40 & Center & Line-15 & 4.2 & 2.9 & 12.8 \\
RPC-based & 60 & Center & Line-15 & 4.8 & 3.3 & $\mathbf{2 0 . 3}$ \\
Crystal-based & - & Center & Point & 30.2 & $\mathbf{2 1 . 6}$ & 45.9 \\
RPC-based & 20 & Center & Point & 2.8 & 1.9 & 5.0 \\
RPC-based & 40 & Center & Point & 5.2 & 3.6 & 13.5 \\
RPC-based & 60 & Center & Point & 5.9 & 4.1 & $\mathbf{2 1 . 4}$ \\
\hline
\end{tabular}

modules (Watts 2014). The trend for off-center sources (results not shown here) is similar but the sensitivity gains are reduced. The improved RPC design with $150 \mu \mathrm{m}$ glass thickness and $2.05 \mathrm{~mm}$ air gap has higher sensitivity compared to the standard RPC prototype. For the configurations with 60 stacks the increase in sensitivity from the standard to the improved RPC-based scanner is a factor 6 and 5 for the $15 \mathrm{~cm}$ line source and point-like sources at the center, respectively. The sensitivity obtained for the RPC-based system with 60 stacks and the line source is on average 2-3 times smaller compared to crystal-based systems. For the point source, the sensitivity is one order of magnitude smaller for the full-ring and standard partialring, and 3-4 times smaller for improved partial-ring configuration. However, if we compare the standard crystal-based system with the improved RPC-based scanner (indicated in bold in table 5), the sensitivity is higher in the case of the line source for the RPC.

3.2.2. Spatial resolution. The spatial resolution for full- and partial-ring configurations are presented in table 6 for a point source at a central and off-center position. The simulated data of the point-like sources are reconstructed according to the NEMA protocol. When comparing the spatial resolution values for the crystal-based systems, we observe that the values are $1 \mathrm{~mm}$ better than those published by Surti et al (2007). Among other reasons we can identify the individual crystal readout that leads to a better spatial resolution.

Depending on the design of the electrode configuration for RPC-based systems, the latter and crystal-based systems may have similar spatial resolution in radial and tangential directions. The RPC-based system has reduced spatial resolution in the axial direction, expected from experimental measurements $(4 \mathrm{~mm})$. In the case of the crystal-based systems, the crystal sizes in the axial and the tangential dimensions are equal. In both technologies, a tangential degradation of the spatial resolution for the partial-ring configurations was observed due to the missing angular sampling.

3.2.3. Scatter fraction. The measured SF of crystal-based system with standard geometry is $32.9 \%$, which is slightly larger than that published by Surti et al (2007), yet it is still acceptable in validation. Scatter fraction values for RPC-based and crystal-based scanners with different configurations are shown in table 7 . In the assessment of the scatter fraction, only the scattered events produced the phantom are considered.

Results in table 7 show that the RPC-based system has about two times higher SF than the crystal-based system. This result is expected since the RPC-based system has no energy 
Table 6. Spatial resolution in radial (R), tangential (T), axial (A) directions in $\mathrm{mm}$ for crystal-based and RPC-based systems.

\begin{tabular}{|c|c|c|c|c|c|}
\hline \multirow[b]{3}{*}{ Scanner } & \multirow[b]{3}{*}{ Stacks } & \multirow[b]{3}{*}{ Position } & \multirow{3}{*}{$\frac{\text { Full-ring }}{\mathrm{R}, \mathrm{T}, \mathrm{A}}$} & \multicolumn{2}{|c|}{ Partial-ring } \\
\hline & & & & std & ext/imp \\
\hline & & & & $\mathrm{R}, \mathrm{T}, \mathrm{A}$ & $\mathrm{R}, \mathrm{T}, \mathrm{A}$ \\
\hline Crystal-based & - & Center & $3.3,3.0,3.1$ & $3.1,3.2,3.3$ & $3.2,3.2,3.6$ \\
\hline RPC-based & 60 & Center & $3.7,3.7,6.6$ & $3.5,4.2,6.5$ & $3.5,4.2,6.9$ \\
\hline Crystal-based & - & $10 \mathrm{~cm}$ offset & $3.7,3.4,3.2$ & $3.5,4.2,3.2$ & $3.6,4.0,3.5$ \\
\hline RPC-based & 60 & $10 \mathrm{~cm}$ offset & $3.8,3.9,7.1$ & $3.6,4.6,7.0$ & $3.4,4.6,7.0$ \\
\hline
\end{tabular}

Table 7. Scatter fraction of the simulated systems.

\begin{tabular}{llll}
\hline & & \multicolumn{2}{c}{ Partial ring } \\
\cline { 3 - 4 } & Full-ring (\%) & Standard (\%) & Extended/Improved (\%) \\
\hline Crystal-based & 32.9 & 32.5 & 32.8 \\
RPC-based with 20 stacks & 61.4 & 58.0 & 60.3 \\
RPC-based with 40 stacks & 60.3 & 56.2 & 57.6 \\
RPC-based with 60 stacks & 60.9 & 56.6 & 56.9 \\
\hline
\end{tabular}

information and therefore the use of an energy window to remove scatter events is not possible. In addition, the axial size of the RPC-based system is larger than the crystal-based systems. For the same reason, when comparing the standard with the extended/improved proposals of partial-ring scanners for the same technology, the increase of the axial size leads to an increase in the scatter fraction. An interesting effect was observed: the reduction of the scatter fraction when the number of stacks is incremented. In Blanco et al (2003) it is shown that the quantum efficiency scales linearly with energy higher than $100 \mathrm{keV}$ when a large number of stacks is being used. In this way, the RPC-based scanner with more stacks is more sensitive to $511 \mathrm{keV}$ photons than scattered photons with less energy, producing a reduction in the scatter fraction. Nevertheless, even for a larger number of stacks the scatter fraction of the RPC-based scanners is higher than crystal-based (by almost a factor 2), which may reduce the quality of the images and there is no standard correction method for scatter events in RPC-based scanners. All the standard correction methods are based on the energy information, which in the case of the RPC technology is not available. Nevertheless, before developing a scatter correction method, it would be necessary to study first the image degradation due to the scatter events inclusion since the RPC technology is energy sensitive. This is out of the scope of the paper.

\subsection{Proton pencil beams}

The reconstructed images of the proton pencil beams are shown in the first row of figure 5 for the full- and partial-ring of the standard crystal-based system. In these images the artifacts created by the missing projections are observed. Improvement in TOF resolution (lowering CRTs) helps compensate for such artifacts, although they are not removed completely. For the case of the RPC-PET, the reconstructed images of the standard system are presented in the first three rows of figure 6, where in addition to the different CRT values, the number of stacks in the RPC scanner is also varied. As previously, the artifacts appear due to the partial-ring arrangement, and increasing TOF resolution reduces such artifacts. RPC images are noisier than crystal-based ones due to the low sensitivity of the RPC technology, even in the case of 
Crystal-based PET
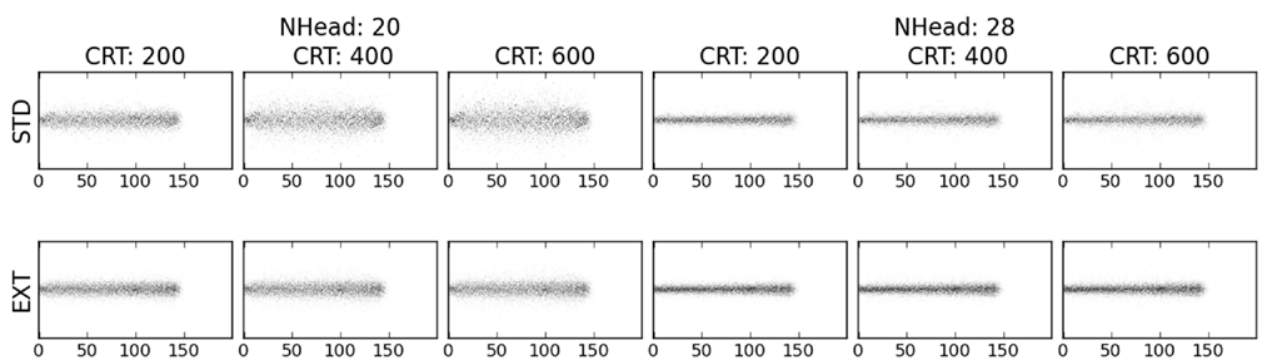

Figure 5. Crystal-based PET reconstructed images of the simulated positron distributions generated by the proton beams. First row: standard configuration. Second row: extended configuration. First three columns: partial-ring geometry (20 heads). Last three columns: full-ring geometry ( 28 heads). The reconstructed images of the same column are obtained using the same CRT value (in $\mathrm{ps}$ ). $5 \cdot 10^{7}$ protons shot on the target. The axes are in $\mathrm{mm}$.

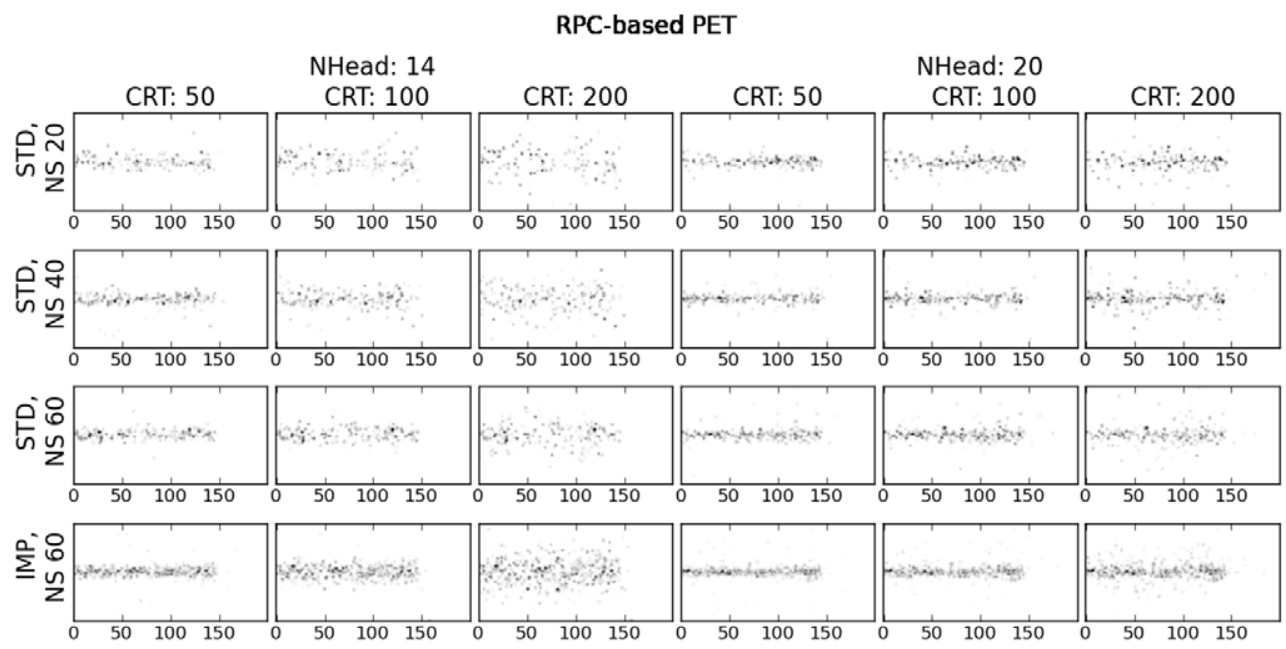

Figure 6. RPC-based PET reconstructed images of the simulated positron distributions generated by the proton beams and acquired by the partial-ring ( 14 heads) and full-ring (20 heads). First three rows: standard configuration, each row corresponds to a number of stacks (NS). Last row: improved scanner for the 60 stacks. The reconstructed images of the same column are obtained using the same CRT value (in ps). $5 \cdot 10^{7}$ protons shot on the target. The axes are in $\mathrm{mm}$.

the greatest number of stacks. The reconstructed images of the positron distribution generated by the pencil beams when using the extended crystal-based and the improved RPC-based PET scanners are shown in the last rows of figures 5 and 6, respectively. For full-ring geometry, there are no noticeable differences between these images and the corresponding images of the standard-configuration systems, despite the increase in sensitivity and angular sampling. This behavior is due to the reduced axial extension of the sources used (less than $1 \mathrm{~cm}$ ). A visible improvement is expected to be observed for more extended source in all dimensions, like in the following section when reconstructing patient data. In the case of the partial-ring geometries, the reconstructed images of the extended and improved configurations are closer 

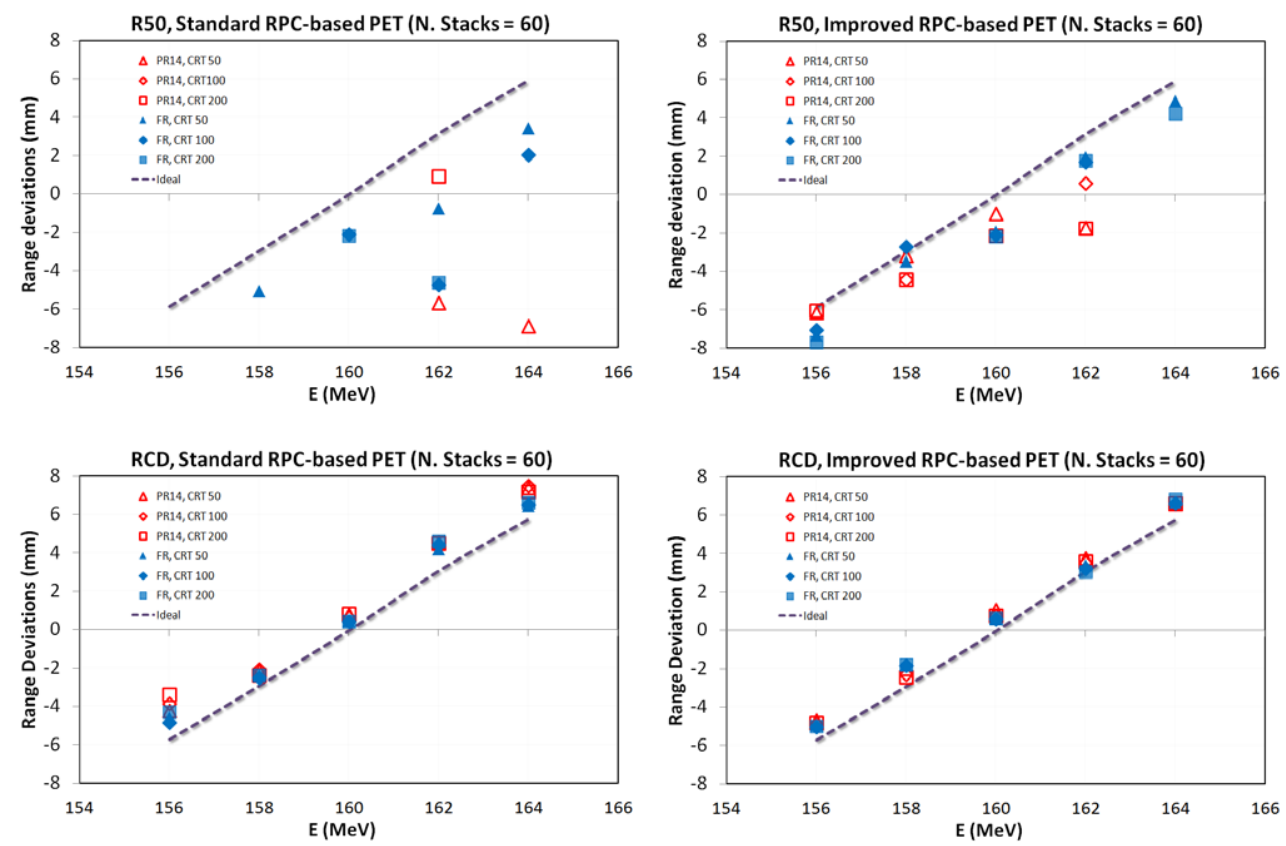

Figure 7. Distal edge differences calculated using $R 50$ (upper row) and $R C D$ (lower row) assessed from a distal edge of the original distribution at $160 \mathrm{MeV}$ protons. The reconstructed images correspond to the simulated data of the RPC-based PET geometries of 60 stacks per head for different CRTs values and for full-ring (FR) and partial-ring (PR) configurations.

to those obtained from the full-ring, so the axial extension seems to compensate for the missing radial detector heads.

The assessment of range deviations from the distributions are shown in figure 7 for the $R 50$ and the $R C D$ of the RPC-based geometries with 60 stacks. When calculating the distal edge of the different distributions and comparing to a reference value (160 MeV protons), ideally we should obtain a straight line with slope of approximately $1.5 \mathrm{~mm} \mathrm{MeV}^{-1}$. However, this slope was not obtained in the case of the standard geometry for the RPC (left-most plot, top row, $R 50$, figure 7), since all the configurations underestimates the range (lower than $-8 \mathrm{~mm}$ ). In the improved RPC geometry, although it is not fully linear, it is notably better. No improvement was observed when introducing better CRT values. When using the RCD (lower row), the expected relationship was observed for the studied energy values for both geometries (standard and improved) and all CRTs. Therefore, when evaluating the capability of the RPCbased PET to detect range deviations of the order of $3 \mathrm{~mm}$, despite the geometry and CRT, the $R C D$ is clearly better suited.

In the case of the crystal-based PET, range differences are presented in figure 8. The main difference compared to the plots for the RPC is that the expected linear relationship with the $1.5 \mathrm{~mm} \mathrm{MeV}{ }^{-1}$ slope is attained for the standard geometry in full-ring and partial-ring configuration of crystal-based PET (left) using the $R 50$ value. When using the extended version, the improvement is clear since the distal edge difference estimations for the partial-ring configuration and for the full-ring for all cases match. For the distal edge estimations, there is no clear effect from the improvement in time resolution. Only the increase in sensitivity and 

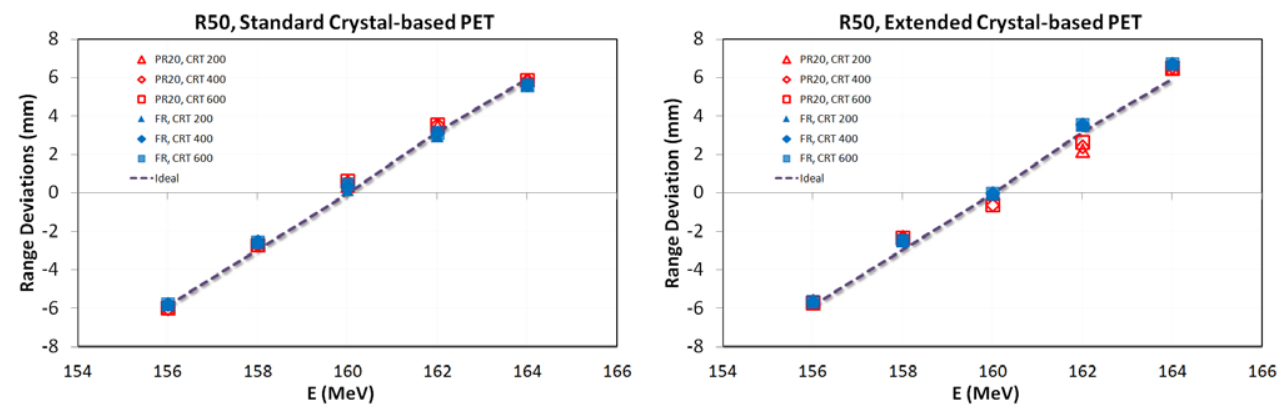

Figure 8. Distal edge differences calculated using $R 50$ obtained from a distal edge of the original distribution at $160 \mathrm{MeV}$ protons. The reconstructed images correspond to the simulated data of the crystal-based PET geometries for different CRTs values and for full-ring (FR) and partial-ring (PR) configurations.

angular sampling seem to play an important role. Similar results were obtained when using the $R C D$. We have calculated the difference between the distal edge estimation of the reconstructed images to the distal edge of the original distribution for the corresponding energy for the standard and extended crystal-based PET and this error is less than $1 \mathrm{~mm}$ for all cases.

We have calculated the goodness of the linear fit of these data for the RPC-based and for the crystal-based PET systems by means of the coefficient of determination $\left(r^{2}\right)$. The low $r^{2}$ values (from 0.132 to 0.709 ) discourage the use of $R 50$ when working with RPC reconstructed data. In the case of crystal-based data, the $r^{2}$ values are acceptable $\left(r^{2}>0.982\right)$ for both magnitudes $(R 50$ and $R C D)$ in all the configurations, even for the standard partial-ring configuration. Although it is not valid for all cases, there is a tendency for higher $r^{2}$ when using lower CRT values. This behavior reproduces observations from reconstructed images of the activity distribution produced by individual beam data, where the improved CRT values reduce the effect of the partial-ring geometry.

\subsection{Patient data}

In this part of the study, we will focus only on the partial-ring configuration since our goal is to evaluate PET systems for on-line dose monitoring. In addition, with regard to the RPC technology, the improved system with 60 stacks presents better characteristics and could perform similarly to the standard crystal system. Therefore, in the following, we will compare the improved design of RPC with the standard and extended configurations of the crystal-based system. The reconstructed images of the patient are shown in figure 9 for the standard and extended crystal-based systems and for the improved RPC-based system in partial-ring configuration. The simulated annihilation points are used as a visual reference figure $9(a)$ for the comparison of reconstructed images with different CRTs. It is important to point out that the axial activity distribution of the patient is larger than the FOV of the standard crystal-based system, although the treatment area is smaller. This is due to the entrance point, the direction of the treatment field and the fragment tail of the carbon-ion beam. The number of annihilations obtained in the simulated irradiation was $3.7 \cdot 10^{5}$ for this patient.

From the images figures $9(b)-(d)$, it was observed that the reconstructed distribution did not reproduce the original activity at the edges of the image. This was due to the size of the axial FOV for the standard crystal-based system in comparison to the extension of the activity distribution. The treatment area is observed clearly at the expected location. In the case of the 


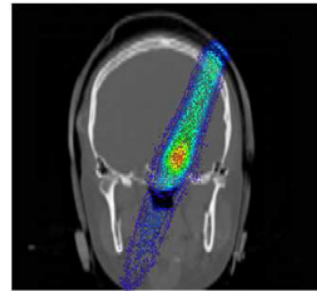

(a) Act. dist. (ref.)

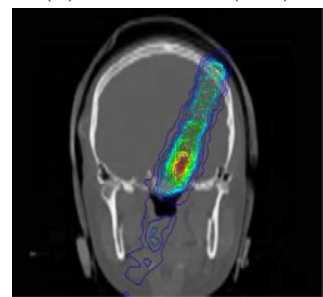

(e) Ext. Crys., No TOF

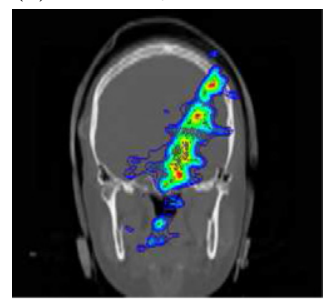

(i) Imp. RPC, No TOF
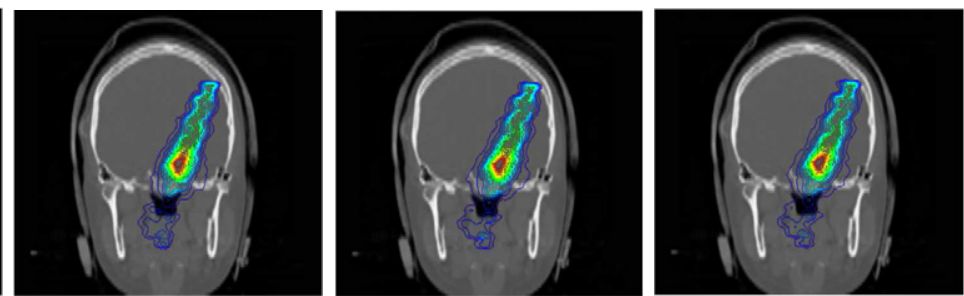

(b) Std. Crystal, $200 \mathrm{p}$
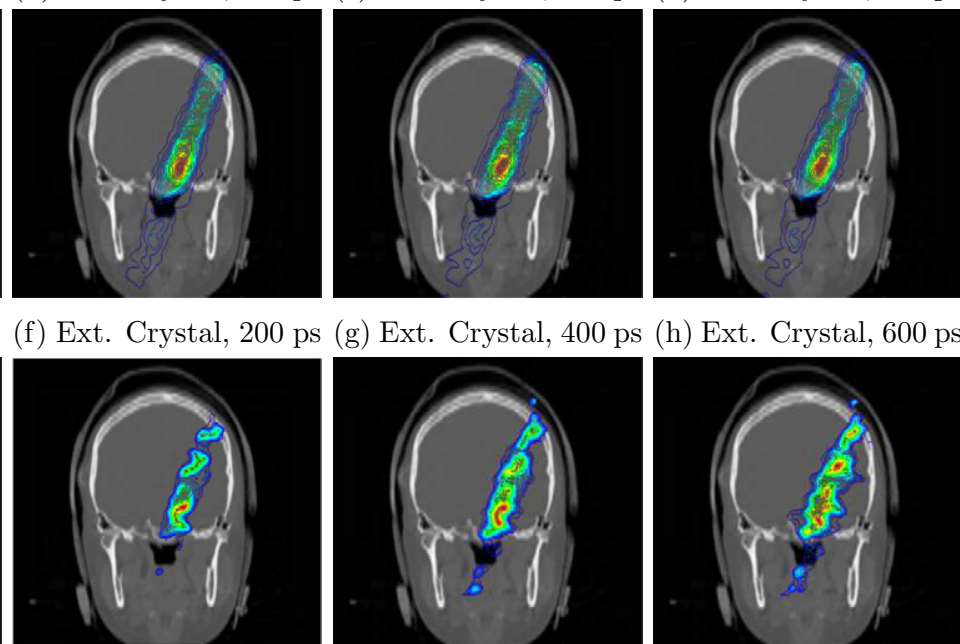

(j) Imp. RPC, $50 \mathrm{ps}$

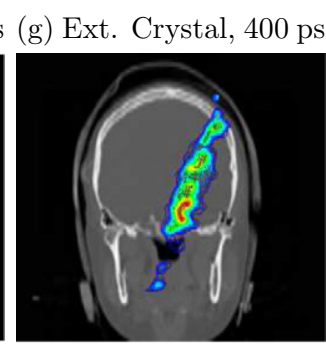

(k) Imp. RPC, $100 \mathrm{ps}$

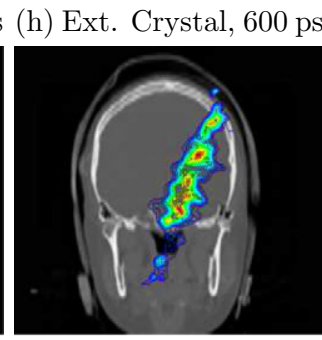

(l) Imp. RPC, 200 ps

Figure 9. Reference activity distribution and reconstructed images of the patient for partial-ring geometries (standard and extended of crystal-based and improved of RPCbased systems) superimposed on the coronal views of the planning CT. Top-left image: the annihilation points distribution of this patient. First row: reconstructed images of the standard crystal-based partial-ring system for different CRTs. Second row: reconstructed images using data of the extended crystal-based partial-ring system changing the CRT values. The most-left image of this row is the reconstructed image without using TOF information. Last row: reconstructed images obtained from an improved RPC-based partial-ring system for different CRTs. As in the second row, the most-left image is reconstructed without including TOF. (a) Act. dist. (ref.). (b) Std. Crystal, 200 ps. (c) Std. Crystal, 400 ps. (d) Std. Crystal, 600 ps. (e) Ext. Crys., No TOF. ( $f$ ) Ext. Crystal, 200 ps. (g) Ext. Crystal, 400 ps. (h) Ext. Crystal, 600 ps. (i) Imp. RPC, No TOF. (j) Imp. RPC, 50 ps. (k) Imp. RPC, 100 ps. (l) Imp. RPC, 200 ps.

extended crystal-based system figures $9(f)-(h)$, the location of treatment area is also correctly reproduced, the edges of the reconstructed activity are coincident with that simulated. With regard to the improved RPC-based PET images figures $9(j)-(l)$, it is difficult to determine where the hot central region is in order to locate the treatment area. When looking at the image figure $9(i)$, for which no TOF information was used for the reconstruction, truncation artifacts (reconstructed activity outside the irradiated area) are visible which disappear when the TOF is included. For the different CRTs, no refinement is perceived in the improved RPC-based PET images, only the reduction of truncation artifacts when TOF is used. For the crystal-based images, the reduction in CRT has a small impact on the image quality.

The profiles obtained along the beam direction from reconstructed images of crystal-based and RPC-based systems are shown in figure 10. In all plots the profile of the annihilation 


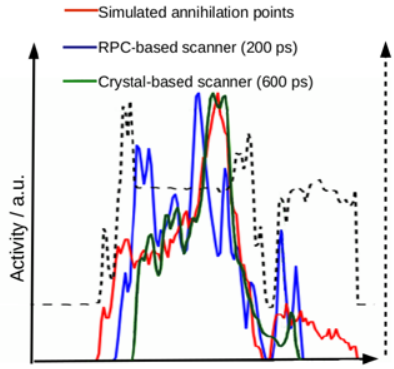

Beam direction

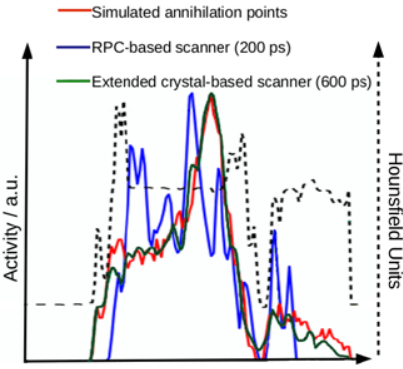

Beam direction

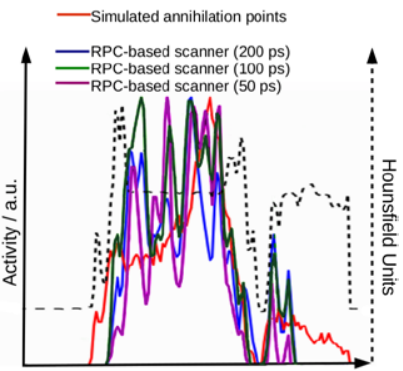

Beam direction

Figure 10. Profiles along the beam direction of the patient in the case of the partialring geometries for standard crystal-based, extended crystal-based and improved RPC-based PET. The red line shows the annihilation points and the dashed black line indicates the Hounsfield units along the profile. Left and central plot: the green line is the reconstructed image for crystal-based (standard or extended) and $600 \mathrm{ps}$ and the blue line is for the RPC data with 200 ps. Right plot: the blue, green and purple lines correspond to 200, 100, 50 ps CRT, respectively, of the improved RPC-based PET.

points distribution is shown as a reference. The profile of the Hounsfield units along the beam direction is also presented for anatomical information. These plots were generated with a tool designed for automatic range assessment (Helmbrecht et al 2012).

In figure 10, profiles of the images corresponding to the improved RPC-based and the crystal-based systems are shown using the original activity distribution as reference. For the two most-left plots, it is clearly seen that the crystal-based systems provide images which are closer to the original activity distribution than the improved RPC-based system. The reconstructed images that reproduce the annihilation points distribution most reliably are those from the extended crystal-based scanner. The reconstructed images of the standard crystalbased system are inferior to the extended system, but still outperform the reconstructed images from the improved RPC-based system.

In order to visualize the enhancement of using better time resolution in the RPC-based system, we have calculated profiles (right plot of figure 10) from the improved RPC-based system for the different CRT values studied. As it can be seen, the profiles of the reconstructed activity are narrower than the original annihilation distribution and the use of a better TOF does not get closer to the original distribution (as one would expect in order to determine at least the edges of the measured activity distribution).

\section{Discussion and conclusions}

In the present work, we investigated the performance of an RPC-based PET system for range verification in particle therapy. Performance characteristics of the RPC-based PET was compared with geometries of a commercially available crystal-based PET and its use for in-beam monitoring in realistic scenarios was studied. Results showed that, in general, the RPC-based PET had lower sensitivity compared to crystal-based PET when we compared with the same configuration (standard versus standard or extended versus improved). It was observed that for extended and centered sources, the improved RPC-based scanner shows higher sensitivity than the standard crystal-based system. Spatial resolution of the system is similar to crystalbased, except in the axial direction because of the intrinsic resolution of the RPC in this direction. The scatter fraction of the RPC-based system was higher due to the larger axial FOV and the lack of energy information. 
The capabilities of these technologies to detect range shifts was studied by modifying the energy of the simulated proton beam. It was observed that both technologies could detect a 3 $\mathrm{mm}$ difference, but a special noise-robust figure-of-merit was required when using the RPCbased scanners. For the case of the RPC-based PET, it was necessary to use the cumulative distribution $(R C D)$ in order to reduce the noise from the reconstructed images, due to the low sensitivity. In the case of the crystal-based PET, the calculation of the $R 50$ was sufficient to differentiate range shifts of $3 \mathrm{~mm}$. For distal edge estimation, no clear benefit was observed when improving the CRT of the simulated systems. In the reconstructed images of the partialring geometries the advantage of using low CRT values is evident since it reduces the scattered data and concentrates the activity along the beam line.

Both technologies were compared regarding patient data of carbon-ion treatments. The quality of the images reconstructed from RPC-technology data was poor and did not provide the expected distribution, assessed using 2D slices and in 1D profiles. The reconstructed images of the crystal-based systems are similar to the original distribution in both the 2D slices and the profile. It should be noted that carbon-ion treatments is a more challenging scenario, since the number of positron emitting isotopes produced during the irradiation is lower than for the proton-beam therapy.

In order to overcome the reduced sensitivity of the RPC technology, systems with increased solid angle could be designed. However for in-beam monitoring, the size of the scanner is constrained by the gantry and large devices may not fit in the treatment room. In addition, considering that treatment field sizes are, at maximum, $20 \times 20 \times 20 \mathrm{~cm}^{3}$ further increasing the axial dimension will not provide the same sensitivity of the crystal-based technology, even in standard configurations.

The reconstruction algorithm that was employed in this study is a standard list-mode MLEM. It was demonstrated in Cabello et al (2013) that including total variation regularization in the cost function produces images with low noise properties without degrading the spatial resolution. This could be a promising approach when handling with RPC data to reduce noise from the reconstructions, it will also enhance crystal-based systems.

Despite its excellent time resolution and the possibility of building large area detectors at low cost, the low sensitivity of the RPC-based scanner limits its use for in-beam monitoring of hadron-therapy. If the RPC technology wants to play a role in this field, efforts in increasing the detector efficiency should be made. One approach may be to reduce the detector diameter, this would not imply any reduction of the spatial resolution since the technology provides DOI in its layered arrangement. This reduction in size should be studied while keeping in mind patient comfort. Other possible enhancements could be the use of a more efficient material for the glass layer. Although this study has investigated the limitations of the RPC technology for on-line dose monitoring in particle therapy, this does not imply that these devices could not be useful for other applications like whole-body PET diagnostic imaging (Blanco et al 2009). In the latter, the possibility of performing a whole body acquisition with only one bed position is a big advantage. However in activity-limited scenarios for which system expansion is constrained, a higher-sensitivity device, such as crystal-based systems, is preferred.

\section{Acknowledgments}

This work was supported by the EU FP7 project ENVISION (Grant Agreement Nr 241851), by the ITN ENTERVISION (FP7, G.A. No. 264552), by Spanish Ministerio de Economía y Hacienda, through 'Juan de la Cierva program', FPA2010-14891 grant, FIS2011-14585, and 
PTA2011-6139-I, by the Generalitat Valenciana through GV/2013/133, and the University of Valencia trough UV-INV-PRECOMP12-80755. Finally the authors would like to acknowledge the GRID-CSIC Spanish project (ref. 200450E494).

\section{References}

Agostinelli S et al 2003 GEANT4-a simulation toolkit Nucl. Instrum. Methods Phys. Res. A 506 250-303 Allison J et al 2006 Geant 4 developments and applications IEEE Trans. Nucl. Sci. 53 270-8

Auffray E et al 2013 A comprehensive and systematic study of coincidence time resolution and light yield using scintillators of different size and wrapping IEEE Trans. Nucl. Sci. $603163-71$

Blanco A, Chepel V, Ferreira-Marques R, Fonte P, Lopes M I, Peskov V and Policarpo A 2003 Perspectives for positron emission tomography with RPCs Nucl. Instrum. Methods A 508 88-93

Blanco A, Couceiro M, Crespo P, Ferreira N C, Ferreira-Marques R, Fonte P, Lopes L and Neves J A 2009 Efficiency of RPC detectors for whole-body human TOF-PET Nucl. Instrum. Methods A 602 780-3

Cabello J, Torres-Espallardo I, Gillam J E and Rafecas M 2013 PET reconstruction from truncated projections using total-variation regularization for hadron therapy monitoring IEEE Trans. Nucl. Sci. 60 3364-72

Crespo P, Shakirin G and Enghardt W 2006 On the detector arrangement for in-beam PET for hadron therapy monitoring Phys. Med. Biol. 51 2143-63

Crespo P, Reis J, Couceiro M, Blanco A, Ferreira N C, Ferreira Marques R, Martins P and Fonte P 2012 Whole-body single-bed time-of-flight RPC-PET: simulation of axial and planar sensitivities with NEMA and antropomorphic phantoms IEEE Trans. Nuc. Sci. $59520-9$

Conti M 2009 State of the art and challenges of time-of-flight PET Phys. Med. 25 1-1

Enghardt W, Crespo P, Fiedler F, Parodi K, Pawelke J and Pönisch F 2004 Charged hadron tumour therapy monitoring by means of PET Nucl. Instrum. Methods A $\mathbf{5 2 5} 284-8$

Groiselle C J and Glick S J 2004 3D PET list-mode iterative reconstruction using time-of-flight information IEEE Nucl. Sci. Symp. Conf. Record pp 2633-8

Hättner E, Iwase H, Krämer M, Kraft G and Schardt D 2013 Experimental study of nuclear fragmentation of 200 and $400 \mathrm{MeV} \mathrm{u}^{-1}{ }^{12} \mathrm{C}$ ions in water for applications in particle therapy Phys. Med. Biol. $588265-79$

Helmbrecht S, Santiago A, Enghardt W, Kuess P and Fiedler F 2012 On the feasibility of automatic detection of range deviations from in-beam PET data Phys. Med. Biol. 57 1387-97

Jan S et al 2011 GATE V6: a major enhancement of the GATE simulation platform enabling modelling of CT and radiotherapy Phys. Med. Biol. 56 881-901

Karp J, Surti S, Daube-Witherspoon M E and Muehllehner G 2008 Benefit of time-of-flight in PET: experimental and clinical results J. Nucl. Med. 49 462-70

Knopf A and Lomax A 2013 In vivo proton range verification: a review Phys. Med. Biol. 58 R131-60

Laukner K 1999 Entwicklung eines iterativen 3D Rekonstruktionsverfahrens für die Kontrolle der Tumorbehandlung mit Schwerionen mittels Positronen-Emissions-Tomographie Dr-Ing. Thesis Technische Universität Dresden

Min C H, Zhu X, Winey B A, Grogg K, Testa M, El Fakhri G, Bortfeld T R, Paganetti H and Shih H A 2013 Clinical application of in-room positron emission tomography for in vivo treatment monitoring in proton radiation therapy Int. J. Radiat. Oncol. Biol. Phys. $86183-9$

National Electrical Manufacturers Association 2001 NEMA Standards Publication NU 2-2001 Performance Measurements of Positron Emission Tomographs (Rosslyn, VA: National Electrical Manufacturers Association)

Nishio T, Takashi O, Kazuhiro N and Hiroshi U 2006 Dose-volume delivery guided proton therapy using beam on-line PET system Med. Phys. 33 4190-7

Oelfke U, Lam G K Y and Atkins M S 1996 Proton dose monitoring with PET: quantitative studies in Lucite Phys. Med. Biol. 41 177-96

Paganetti H 2012 Range uncertainties in proton therapy and the role of Monte Carlo simulations Phys. Med. Biol. 57 R99-117

Parodi K and Bortfeld T 2006 A filtering approach based on Gaussian-powerlaw convolutions for local PET verification of proton radiotherapy Phys. Med. Biol. 51 1991-2009 
Parodi K et al 2007 Patient study of in vivo verification of beam delivery and range, using positron emission tomography and computed tomography imaging after proton therapy Int. J. Radiat. Oncol. Biol. Phys. 68 920-34

Pönisch F, Parodi K, Hasch B G and Enghardt W 2004 The modelling of positron emitter production and PET imaging during carbon ion therapy Phys. Med. Biol. 49 5217-32

Priegnitz M, Möckel D, Parodi K, Sommerer F, Fiedler F and Enghardt W 2008 In-beam PET measurement of ${ }^{7} \mathrm{Li}^{3+}$ irradiation induced $\beta^{+}$-activity Phys. Med. Biol. $534443-53$

PTCOG 2014 (http://ptcog.web.psi.ch/ptcentres.html)

Remmele S, Hesser J, Paganetti H and Bortfeld T 2011 A deconvolution approach for PET-based dose reconstruction in proton radiotherapy Phys. Med. Biol. 56 7601-19

Shakirin G 2009 System solution for in-beam positron emission tomography monitoring of radiation therapy $P h D$ Thesis Technische Universität Dresden

Shakirin G, Crespo P, Fiedler F, Wagner A and Enghardt W 2008 Optimum voxel size for reconstruction of in-beam PET data IEEE Nucl. Sci. Symp. Conf. Record pp 5066-9

Shakirin G, Bräss H, Fiedler F, Kunath D, Laube K, Parodi K, Priegnitz M and Enghardt W 2011 Implementation and workflow for PET monitoring of therapeutic ion irradiation: a comparison of in-beam, in-room and off-line techniques Phys. Med. Biol. 56 1281-98

Siddon R L 1985 Fast Calculation of the exact radiological path for a three-dimensional CT array Med. Phys. 12 252-5

Surti S, Kuhn A, Werner M E, Perkins A E, Kolthammer J and Karp J S 2007 Performance of Philipps Gemini TF PET/CT scanner with special consideration for its time-of-flight imaging capabilities J. Nucl. Med. 48 471-80

Surti S, Zou W, Daube-Witherspoon M E, McDonough J and Karp J S 2011 Design study of an in situ PET scanner for the use in proton beam therapy Phys. Med. Biol. 56 2667-85

Tashima H, Yamaya T, Yoshida E, Kinouchi S, Watanabe M and Tanaka E 2012 A single-ring OpenPET enabling PET imaging during radiotherapy Phys. Med. Biol. 57 4705-18

TERA 2013 (http://project-aqua.web.cern.ch/project-aqua)

van Dam H T, Borghi G, Seifert S and Schaart D R 2013 Sub-200 ps CRT in monolithic scintillator PET detectors using digital SiPM arrays and maximum likelihood interaction time estimation Phys. Med. Biol. 58 3243-57

Watts D, Borghi G, Sauli F and Amaldi U 2013 The use of multi-gap resistive plate chambers for in-beam PET in proton and carbon ion therapy J. Radiat. Res. 54 i136-42

Watts D 2014 Detectors for quality assurance in hadrontherapy PhD Thesis Universitat Politècnica de Catalunya, Barcelona

Yamaya T, Inaniwa T, Minohara S, Yoshida E, Inadama N, Nishikido F, Shibuya K, Lam C F and Murayama H 2008 A proposal of an open PET geometry Phys. Med. Biol. 53 757-73 